\title{
O papel do enfermeiro com o cateter central de inserção periférica: revisão integrativa
}

\author{
The role of the nurse with the peripheral inserted central \\ catheter: integrative review
}

\begin{abstract}
Aline Cerqueira Santos Santana da Silva' • Érick Igor dos Santos ${ }^{2}$ Priciana Teixeira Queiroz ${ }^{3}$.
\end{abstract} Fernanda Garcia Bezerra Góes ${ }^{4}$

\begin{abstract}
RESUMO
Objetiva-se analisar as evidências científicas acerca do papel do enfermeiro na utilização do cateter central de inserção periférica (PICC) em unidades de terapia intensiva pediátrica e neonatal. Estudo descritivo, baseado em revisão integrativa da literatura, norteado pela questão: como a prática clínica do enfermeiro com o cateter central de inserção periférica tem sido abordada em unidades de terapia intensiva pediátrica e neonatal segundo evidências cientificas nacionais e internacionais? As buscas foram realizadas nas bases de dados Literatura Latino-Americana e do Caribe em Ciências da Saúde (LILACS), Medical Literature Analysis and Retrieval System Online (MEDLINE) e na Scientific Electronic Library Online (SciELO), por meio da associação dos descritores "cateterismo periférico," "infusões intravenosas", "neonatologia", "pediatria" " "terapia intensiva", no período de outubro a dezembro de 2015. Foram selecionados para a análise crítica 16 artigos, os quais foram divididos em três categorias temáticas: 1- competência técnica e legal como pilar de sustentação na prática com o PICC, 2- conhecimentos, atitudes e práticas do profissional enfermeiro na inserção, manutenção e retirada do PICC e, 3- tomada de decisão na utilização do PICC. Concluí-se que a utilização do PICC na prática clínica do enfermeiro possui recomendações que requerem capacitação e habilitação profissional para a tomada de decisão, a inserção, a manutenção e a retirada, visando a redução de eventos adversos e a manutenção do bem-estar do paciente.

Palavras-chave: Cateterismo Periférico; Infusões Intravenosas; Neonatologia, Pediatria; Terapia Intensiva.
\end{abstract}

\section{ABSTRACT}

The aim is to analyze the scientific evidence about the role of the nurse with the peripheral inserted central catheter in pediatric and neonatal intensive care units. Descriptive study, based on an integrative literature review, guided by the question: how has the clinical practice of the nurse with the peripheral inserted central catheter been approached in pediatric and neonatal intensive care units according to national and international scientific evidence? The searches were carried in the database Latin American and Caribbean Health Sciences (LILACS), Medical Literature Analysis and Retrieval System Online (MEDLINE) e na Scientific Electronic Library Online (SciELO), by means of the association of the descriptors "peripheral catheterization","intravenous infusions","neonatology","pediatrics" and "intensive therapy", between October and December 2015.16 articles were selected for the critical analysis, which were divided into three thematic categories: 1-technical and legal expertise as a pillar of support in practice with the PICC, 2- knowledge, attitudes and practices of nurses in the insertion, maintenance and withdrawal of PICC; 3- decision-making in the use of the PICC. It concludes that the use of PICC in the clinical practice of the nurse has recommendations that require training and professional qualification for decision making, insertion, maintenance and withdrawal, aiming at reducing adverse events and maintaining patient well-being.

Keywords: Peripheral Catheterization; Intravenous Infusions; Neonatology; Pediatrics; Intensive Therapy.

${ }^{1}$ Enfermeira Pediatra pela Universidade Federal do Rio de Janeiro (UFRJ). Doutora e Mestre em Enfermagem pela UFRJ. Professora Assistente da Universidade Federal Fluminense (UFF). Pesquisadora dos Grupos de Pesquisa Laboratório sobre Enfermagem, Cuidado, Inovação e Organização da Assistência ao Adulto ou ao Idoso (LECIONAI) e Estudos sobre Vivências e Integralidade Dedicadas à Enfermagem, Criança, Infância, Adolescentes e Recém-nascidos (EVIDENCIAR) - UFF. Rio das Ostras, RJ, Brasil. E-mail: alinecer2014@gmail.com ${ }^{2}$ Enfermeiro Estomaterapeuta pela Universidade do Estado do Rio de Janeiro (UERJ). Doutor e Mestre em Enfermagem pela UERJ. Professor Assistente da Universidade Federal Fluminense (UFF). Pesquisador dos Grupos de Pesquisa Laboratório sobre Enfermagem, Cuidado, Inovação e Organização da Assistência ao Adulto ou ao Idoso (LECIONAI) e Estudos sobre Vivências e Integralidade Dedicadas à Enfermagem, Criança, Infância, Adolescentes e Recém-nascidos (EVIDENCIAR) - UFF. Rio das Ostras, RJ, Brasil. E-mail: eigoruff@gmail.com

${ }^{3}$ Graduanda de Enfermagem pela Universidade Federal Fluminense (UFF). Estudante do Grupo de Pesquisa Estudos sobre Vivências e Integralidade Dedicadas à Enfermagem, Criança, Infância, Adolescentes e Recém-nascidos (EVIDENCIAR) - UFF. Rio das Ostras, RJ, Brasil. E-mail: pricianatqueirozgmail.com

${ }^{4}$ Enfermeira Pediatra pela Universidade Federal do Rio de Janeiro (UFRJ). Mestre em Enfermagem pela Universidade Federal do Estado do Rio de Janeiro (UNIRIO) e Doutora em Enfermagem pela Universidade Federal do Rio de Janeiro (UFRJ). Professora Adjunta da Universidade Federal Fluminense (UFF). Pesquisadora do Grupo de Pesquisa Estudos sobre Vivências e Integralidade Dedicadas à Enfermagem, Criança, Infância, Adolescentes e Recém-nascidos (EVIDENCIAR)- UFF. Rio das Ostras, RJ, Brasil. E-mail: ferbezerra@gmail.com 


\section{INTRODUÇÃO}

As unidades de tratamento intensivo (UTI) neonatal e pediátrica constituem-se em ambientes terapêuticos apropriados para a recuperação e a sobrevida de pacientes em estado crítico. Nestes cenários, um dos maiores desafios para o sucesso da terapêutica implementada é a manutenção de um acesso venoso seguro para esta população.

$\mathrm{Na}$ última década, tem-se observado um crescente número de tratamentos e procedimentos que utilizam acessos vasculares para a administração intravenosa de soluções e drogas ${ }^{1}$.

Nessa corrente, grande êxito tem sido alcançado através da inserção de cateteres centrais inseridos perifericamente, que asseguram a recuperação clínica de pacientes críticos, tal como os cateteres que são inseridos centralmente ${ }^{2}$.

$\mathrm{Na}$ década de 70, nos países Europeus e nos Estados Unidos da América, devido à necessidade de manutenção de acessos venosos prolongados para infusão de quimioterápicos, nutrição parenteral total (NPT), até mesmo no cuidado domiciliar, foi desenvolvido um dispositivo intravenoso, denominado cateter central de inserção periférica (PICC), que inicialmente passou a ser utilizado em unidades de terapia intensiva ${ }^{3}$.

0 cateter de silicone surge no mercado a partir da década de 70 , porém é na década de 80 que se observa um incremento na expansão e implementação da técnica com esse cateter, devido à praticidade de inserção à beira do leito por um enfermeiro e ao surgimento de programas de capacitação profissional ${ }^{4}$. No Brasil, o PICC passa a ser utilizado a partir da década de 90, com grande expansão, graças ao conhecimento de suas indicações ${ }^{5-6}$.

Este cateter se apresenta como uma alternativa segura de acesso intravenoso central de permanência prolongada, pois permite infusões de NPT, drogas com alta osmolaridade, com baixo $\mathrm{pH}$ e vasoativas, além de hidratação venosa (HV) com altas taxas de infusão de glicose (TIG) e fármacos variados que apresentam, em sua composição, propriedades irritantes à camada intima da veia. Além disso, apresenta menor custo e menor taxa de infecção quando comparado ao cateter venoso central (CVC) tunelizado ou cirurgicamente inserido ${ }^{3-7-9}$.

Dentre suas desvantagens, podemos destacar a necessidade de vasos calibrosos e íntegros, disponibilidade de aparato tecnológico de qualidade e serviço de radiologia, uso de barreira máxima de proteção e equipe capacitada e habilitada, além de protocolos e normas institucionais ${ }^{10}$.

Apesar de sua praticidade e segurança, ainda é prevalente o desenvolvimento de oclusão, extravasamento, migração da ponta do cateter e trombose, resultando na remoção não eletiva do mesmo ${ }^{2-8-11}$.

Estudos evidenciam que é frequente a necessidade da indicação desse dispositivo para a assistência prestada na UTI pediátrica e neonatal ${ }^{2-12}$. Isto demanda enfermeiros capacitados e habilitados na prática clínica com o PICC, com prioridade à prevenção e à detecção precoce de eventos adversos relacionados ao uso, visando a segurança e o bem-estar do paciente.

Por este motivo, a realização deste procedimento se configura uma prática especializada, complexa e assegurada no Brasil pela Lei do Exercício Profissional $n^{\circ}$ 7.498 de 25 de junho de 1986, que dispõe, em seu artigo 11 , que cabe privativamente ao enfermeiro os cuidados diretos de enfermagem a pacientes graves e com risco de vida, assim como cuidados diretos de enfermagem de maior complexidade técnica e que exijam conhecimento de base científica e capacidade de tomar decisões imediatas $^{13}$. Ademais a Resolução do Conselho Federal de Enfermagem 258/2001, considerando a competência técnica do enfermeiro, afirma que é lícito a esse profissional a inserção deste dispositivo, mediante a qualificação e/ou capacitação profissional ${ }^{14}$.

Nesta diretiva, pela terapia intravenosa ser amplamente utilizada no cenário hospitalar, torna-se válida toda iniciativa que privilegie procedimentos que reduzam os riscos de complicações nos recém-nascidos e crianças, onde se destaca, nesse momento, o PICC.

Diante do exposto, este trabalho objetivou analisar as evidências científicas acerca do papel do enfermeiro na utilização do cateter central de inserção periférica (PICC) em unidades de terapia intensiva pediátrica e neonatal.

\section{MÉTODO}

Trata-se de um estudo descritivo, pautado na revisão integrativa de literatura, que visa a análise de pesquisas relevantes, a síntese do conhecimento sobre determinada temática e a identificação das lacunas que necessitam ser preenchidas sobre um tema definido e específico ${ }^{15}$.

Para tanto, foram percorridas seis etapas:1-identificação do problema com definição da questão de pesquisa, 2 busca em bases de dados e bibliotecas virtuais por meio de descritores, 3- tabulação dos estudos, 4- leitura individual dos textos completos para a análise crítica em relação à sua aderência ao objetivo desta pesquisa, 5-interpretação dos resultados e 6- síntese do conhecimento ${ }^{15}$.

Para condução do estudo foi elaborada a seguinte questão de pesquisa: como a prática clínica do enfermeiro com o cateter central de inserção periférica tem sido abordada em unidades de terapia intensiva pediátrica e neonatal segundo evidências cientificas nacionais e internacionais?

A partir desse questionamento, foram selecionados, no portal de Descritores das Ciências da Saúde (DeCS), os seguintes descritores: "cateterismo periférico", "infusões intravenosas","neonatologia”,"pediatria" e "terapia intensiva”.

A coleta de dados ocorreu de outubro a dezembro de 2015 nas bases de dados Literatura Latino-Americana e do Caribe em Ciências da Saúde (LILACS), Medical Literature 
Analysis and Retrieval System Online (MEDLINE) e na Scientific Electronic Library Online (SciELO), a partir das diferentes associações entre os descritores selecionados.

Para a seleção das publicações, foram estabelecidos os seguintes critérios de inclusão: artigos em inglês e/ ou português, dos últimos 10 anos, e que respondessem à questão de pesquisa. Foram excluídos os artigos em duplicidade, aqueles cujo link para acesso em texto completo apresentasse erro mediante a tentativa de acesso e os artigos in press ou ahead of print, o que inviabilizaria o seu acesso em formato final.

Os dados dos estudos selecionados foram tabulados no software Microsoft Excel 2010 para organização e sumarização das principais informações:título,periódico,ano de publicação, autores, delineamento, resultados/desfecho.

\section{RESULTADOS}

Identificou-se o total de 1321 artigos em todas as bases de dados, dos quais 13 estavam em duplicidade. Após a seleção pelos critérios de exclusão foram descartadas 308 publicações. Após a leitura completa do texto, mais artigos foram excluídos pelo fato de não responderem a questão da pesquisa. Assim, a amostra final foi constituída por 16 artigos, como verifica-se na figura 1.

Os artigos selecionados foram publicados entre os anos 2005 a 2015. Houve, em número de publicações por ano, uma publicação nos anos de 2006, 2008 e 2013, duas publicações nos anos de 2007, 2009 e 2010, três publicações no ano de 2012 e, em destaque, quatro publicações no ano de 2011. Observa-se, assim, que o maior número de publicações se concentrou nos anos entre 2011 e 2012.

Considerando o local de publicação, oito publicações são nacionais, sendo: dois estudos publicados na região sudeste, três no sul e três no centro-oeste. Quanto à publicação internacional, foram encontrados oito artigos, sendo: um artigo publicado na Suíça, um na Irlanda, um na Alemanha, um nos Estados Unidos da América, dois na Holanda e dois na Inglaterra. Dos 16 artigos selecionados, 14 foram desenvolvidos no âmbito da neonatologia.

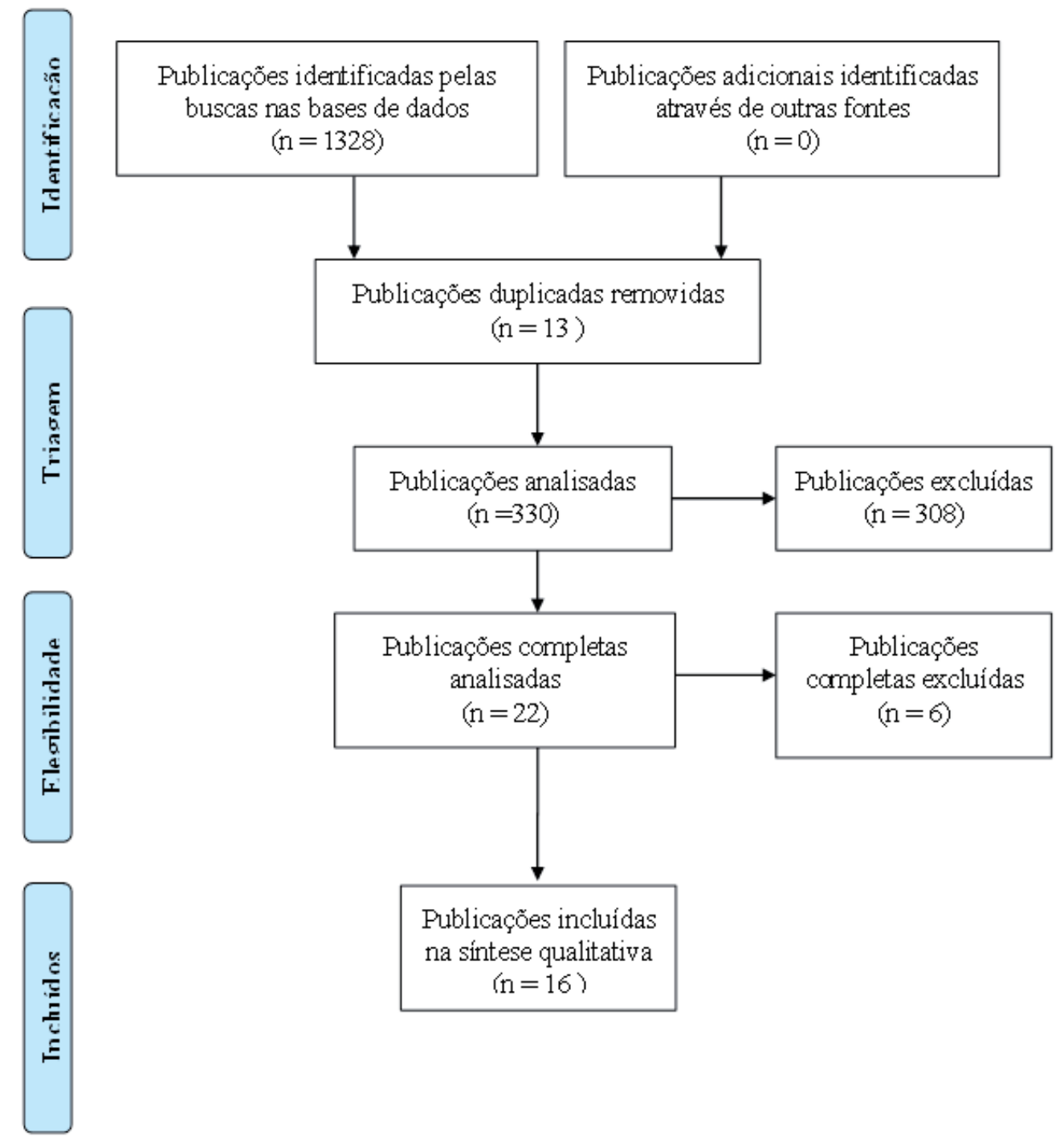

Figura 1: Diagrama de seleção dos artigos incluídos na revisão.

Fonte: elaboração dos autores. 
Após leitura crítica dos estudos foram evidenciadas três categorias temáticas, a saber: 1- competência técnica e legal como pilar de sustentação na prática com o PICC,
2- conhecimentos, atitudes e práticas do profissional enfermeiro na inserção, manutenção e retirada do PICC e, 3- tomada de decisão na utilização do PICC.

Quadro 1. Distribuição dos artigos acerca da prática clínica com o cateter central de inserção periférica no período de 2005 a 2015.

\begin{tabular}{|c|c|c|c|c|}
\hline Título & $\begin{array}{l}\text { Periódico e ano } \\
\text { de publicação }\end{array}$ & Autores & Delineamento & Resultados / Desfecho \\
\hline $\begin{array}{l}\text { An evaluation of peripherally } \\
\text { inserted central venous catheters } \\
\text { for children with cancer requiring } \\
\text { long-term venous access }\end{array}$ & $\begin{array}{l}\text { International } \\
\text { Journal of } \\
\text { Hematology } \\
2011\end{array}$ & $\begin{array}{l}\text { Hatakeyama N; Hori } \\
\text { T; Yamamoto M; } \\
\text { Mizue N }\end{array}$ & $\begin{array}{l}\text { Análise } \\
\text { retrospectiva de } \\
\text { dados }\end{array}$ & $\begin{array}{l}\text { O PICC fornece acesso } \\
\text { intravenoso confiável em } \\
\text { longo prazo em crianças com } \\
\text { doenças malignas. }\end{array}$ \\
\hline $\begin{array}{l}\text { Atuação do enfermeiro no } \\
\text { cuidado com o PICC no recém- } \\
\text { nascido }\end{array}$ & $\begin{array}{l}\text { Revista } \\
\text { Brasileira de } \\
\text { Enfermagem } \\
2006\end{array}$ & $\begin{array}{l}\text { Rodrigues ZS; } \\
\text { Chaves EMC; } \\
\text { Cardoso MVLML }\end{array}$ & $\begin{array}{l}\text { Estudo descritivo } \\
\text { exploratório }\end{array}$ & $\begin{array}{l}\text { O manuseio do PICC requer } \\
\text { conhecimento, destreza e } \\
\text { habilidade por parte dos } \\
\text { enfermeiros e membros da } \\
\text { equipe de saúde. }\end{array}$ \\
\hline $\begin{array}{l}\text { Cateter Central de inserção } \\
\text { Periférica: descrição da utilização } \\
\text { em UTI Neonatal e Pediátrica }\end{array}$ & $\begin{array}{l}\text { Revista da } \\
\text { Escola de } \\
\text { Enfermagem da } \\
\text { USP } \\
2010\end{array}$ & $\begin{array}{l}\text { Baggio MA; Bazzi } \\
\text { FCS; Bilibio CAC }\end{array}$ & $\begin{array}{l}\text { Análise descritiva } \\
\text { retrospectiva }\end{array}$ & $\begin{array}{l}\text { Melhor desempenho } \\
\text { requer capacitação e } \\
\text { educação permanente } \\
\text { dos profissionais, visando } \\
\text { minimizar a remoção } \\
\text { antecipada do cateter. }\end{array}$ \\
\hline $\begin{array}{l}\text { Cateteres Centrais de Inserção } \\
\text { Periférica em crianças de } \\
\text { Hospitais do Município de São } \\
\text { Paulo. }\end{array}$ & $\begin{array}{l}\text { Rev Gaúcha } \\
\text { Enfermagem } \\
2007\end{array}$ & $\begin{array}{l}\text { Vendramim P; } \\
\text { Pedreira MLG; } \\
\text { Peterlini MAS }\end{array}$ & $\begin{array}{l}\text { Estudo descritivo } \\
\text { e de correlação }\end{array}$ & $\begin{array}{l}\text { Há uma diferença } \\
\text { significativa na } \\
\text { predominância do uso } \\
\text { do PICC por instituições } \\
\text { privadas e no setor de } \\
\text { neonatologia. }\end{array}$ \\
\hline $\begin{array}{l}\text { Central Line-Associated } \\
\text { Bloodstream Infection in } \\
\text { Hospitalized Children with } \\
\text { Peripherally Inserted CVC: } \\
\text { Extending Risk Analyses Outside } \\
\text { the Intensive Care Unit }\end{array}$ & $\begin{array}{l}\text { Clinical } \\
\text { Infectious } \\
\text { diseases } \\
2011\end{array}$ & $\begin{array}{l}\text { Advani S; Reich NG; } \\
\text { Sengupta A; Gosey } \\
\text { L; Milstone AM }\end{array}$ & Análise descritiva & $\begin{array}{l}\text { Os fatores associados } \\
\text { à infecção de corrente } \\
\text { sanguínea associada ao } \\
\text { PICC incluíram: idade mais } \\
\text { jovem, doença de base, PICCs } \\
\text { inseridos na extremidade } \\
\text { inferior, tempo prolongado } \\
\text { de permanência do cateter, a } \\
\text { exposição pediátrica à UTI e } \\
\text { administração de NPT. }\end{array}$ \\
\hline $\begin{array}{l}\text { Complicações acerca do cateter } \\
\text { venoso central de inserção } \\
\text { periférica PICC }\end{array}$ & $\begin{array}{l}\text { Cience Cuid } \\
\text { Saúde } \\
2007\end{array}$ & Jesus VC; Secoli SR & $\begin{array}{l}\text { Revisão } \\
\text { Bibliográfica }\end{array}$ & $\begin{array}{l}\text { As complicações } \\
\text { identificadas foram: oclusão, } \\
\text { flebite, mau posicionamento, } \\
\text { sepse, trombose, infecção } \\
\text { local, ruptura, embolização } \\
\text { e dificuldade de remoção do } \\
\text { cateter. }\end{array}$ \\
\hline $\begin{array}{l}\text { Conhecimento de enfermeiros de } \\
\text { neonatologia acerca do Cateter } \\
\text { Venoso Central de Inserção } \\
\text { periférica }\end{array}$ & $\begin{array}{l}\text { Revista } \\
\text { Brasileira de } \\
\text { Enfermagem } \\
2012\end{array}$ & $\begin{array}{l}\text { Belo MPM; Silva } \\
\text { RAMC; Nogueira } \\
\text { ILM; Mizoguti DP; } \\
\text { Ventura CMU }\end{array}$ & $\begin{array}{l}\text { Estudo descritivo } \\
\text { transversal }\end{array}$ & $\begin{array}{l}\text { Profissionais que possuem } \\
\text { habilitação para prática com } \\
\text { o PICC obtiveram melhores } \\
\text { resultados. }\end{array}$ \\
\hline $\begin{array}{l}\text { Hemidiaphragmatic paralysis } \\
\text { in preterm neonates: a rare } \\
\text { complication of peripherally } \\
\text { inserted central catheter } \\
\text { extravasation }\end{array}$ & $\begin{array}{l}\text { Journal of } \\
\text { Pediatric } \\
\text { Surgery } \\
2011\end{array}$ & $\begin{array}{l}\text { Toselloa B; Michela } \\
\text { F; Merrotb T; } \\
\text { Chaumoîtrec K; } \\
\text { Hassida S; Lagiera } \\
\text { P; Martin C }\end{array}$ & Estudo de caso & $\begin{array}{l}\text { Associação entre o } \\
\text { extravasamento de NPT } \\
\text { no tórax no uso do PICC e } \\
\text { complicação respiratória rara. }\end{array}$ \\
\hline $\begin{array}{l}\text { How Long Should Peripherally } \\
\text { Inserted Central Catheterization } \\
\text { Be Delayed in the Context } \\
\text { of Recently Documented } \\
\text { Bloodstream Infection? }\end{array}$ & $\begin{array}{l}\text { Journal of } \\
\text { Vascular and } \\
\text { Interventional } \\
\text { Radiology } \\
2012 \\
\end{array}$ & $\begin{array}{l}\text { Nick Daneman, } \\
\text { MD, MSc, FRCPC, } \\
\text { Mark Downing, MD, } \\
\text { and Brandon M. } \\
\text { Zagorski, MS }\end{array}$ & $\begin{array}{l}\text { Análise de corte } \\
\text { retrospectivo }\end{array}$ & $\begin{array}{l}\text { O risco de bacteremia } \\
\text { recidivante foi maior quando } \\
\text { os PICCs foram inseridos } \\
\text { dentro de } 2 \text { dias do episódio } \\
\text { da bacteremia constatada. }\end{array}$ \\
\hline $\begin{array}{l}\text { Imaging of the complications } \\
\text { of peripherally inserted central } \\
\text { venous catheters }\end{array}$ & $\begin{array}{l}\text { Clinical } \\
\text { Radiology } \\
2009\end{array}$ & $\begin{array}{l}\text { Amerasekera SSH; } \\
\text { Jones CM; Patel R; } \\
\text { Cleasby MJ }\end{array}$ & Estudo de caso & $\begin{array}{l}\text { É importante o papel } \\
\text { do radiologista na } \\
\text { confirmação do adequado } \\
\text { posicionamento do PICC. }\end{array}$ \\
\hline
\end{tabular}


Quadro 1. Continuação

\begin{tabular}{|c|c|c|c|c|}
\hline Título & $\begin{array}{l}\text { Periódico e ano } \\
\text { de publicação }\end{array}$ & Autores & Delineamento & Resultados / Desfecho \\
\hline $\begin{array}{l}\text { Intravenous peripherally-inserted } \\
\text { central catheters for antibiotic } \\
\text { therapy in children with cystic } \\
\text { fibrosis }\end{array}$ & $\begin{array}{l}\text { Journal of Cystic } \\
\text { Fibrosis } 2009\end{array}$ & $\begin{array}{l}\text { Bui S; Babre F; } \\
\text { Hauchecorne S; } \\
\text { Christoflour N; } \\
\text { Ceccato F; Boisserie- } \\
\text { Lacroix V; Clouzeau } \\
\text { H; Fayon M }\end{array}$ & $\begin{array}{l}\text { Estudo descritivo } \\
\text { prospectivo }\end{array}$ & $\begin{array}{l}\text { O PICC é bem tolerado em } \\
\text { crianças com fibrose cística, } \\
\text { sendo um meio eficaz para } \\
\text { a realização da terapia } \\
\text { intravenosa nessa população. }\end{array}$ \\
\hline $\begin{array}{l}\text { Localização Inicial da Ponta } \\
\text { de cateter central de inserção } \\
\text { periférica (PICC) em recém- } \\
\text { nascidos }\end{array}$ & $\begin{array}{l}\text { Revista da } \\
\text { Escola de } \\
\text { Enfermagem da } \\
\text { USP } \\
2008\end{array}$ & $\begin{array}{l}\text { Camargo PP; } \\
\text { Kimura AF; Toma E; } \\
\text { Tsunechiro MA }\end{array}$ & $\begin{array}{l}\text { Estudo } \\
\text { transversal }\end{array}$ & $\begin{array}{l}\text { O mau posicionamento } \\
\text { inicial da ponta do cateter } \\
\text { esteve relacionada à } \\
\text { introdução do comprimento } \\
\text { do cateter além do } \\
\text { necessário. }\end{array}$ \\
\hline $\begin{array}{l}\text { Peripherally inserted central } \\
\text { catheter in leukemia: insertion } \\
\text { site determines clotting risk }\end{array}$ & $\begin{array}{l}\text { Leukemia \& } \\
\text { Lymphoma } 2010\end{array}$ & Periard D. & $\begin{array}{l}\text { Relato de } \\
\text { Experiência }\end{array}$ & $\begin{array}{l}\text { Mudança no protocolo } \\
\text { institucional quanto ao } \\
\text { sítio de inserção do PICC } \\
\text { gerou redução considerável } \\
\text { na incidência de trombose } \\
\text { venosa profunda. }\end{array}$ \\
\hline $\begin{array}{l}\text { Peripherally inserted central } \\
\text { catheter placement in cancer } \\
\text { patients with profound } \\
\text { thrombocytopaenia: a prospective } \\
\text { analysis }\end{array}$ & $\begin{array}{l}\text { European } \\
\text { Journal of } \\
\text { Radiology } 2013\end{array}$ & $\begin{array}{l}\text { Potet J; Thome A; } \\
\text { Curis E; Arnaud } \\
\text { FX; Donat GW; } \\
\text { Valbousquet L; } \\
\text { Peroux E; Flor E; } \\
\text { Dody C; Konopacki } \\
\text { J; Malfuson JV; } \\
\text { Cartry C; Lahutte M; } \\
\text { Revel T; Baccialone } \\
\text { J; Teriitehau CA }\end{array}$ & $\begin{array}{l}\text { Análise } \\
\text { prospectiva }\end{array}$ & $\begin{array}{l}\text { Sucesso na escolha } \\
\text { do PICC como acesso } \\
\text { venoso em pacientes com } \\
\text { trombocitopenia grave. }\end{array}$ \\
\hline $\begin{array}{l}\text { Práticas de manejo do PICC em } \\
\text { uma unidade neonatal }\end{array}$ & $\begin{array}{l}\text { Revista } \\
\text { Brasileira de } \\
\text { Enfermagem } \\
2011\end{array}$ & $\begin{array}{l}\text { Dórea E; Castro } \\
\text { TE;Costa P; Kimura } \\
\text { AF; Santos FMG }\end{array}$ & $\begin{array}{l}\text { Estudo descritivo } \\
\text { exploratório }\end{array}$ & $\begin{array}{l}\text { Procedimentos adotados } \\
\text { na manutenção do PICC } \\
\text { diferem dos descritos no } \\
\text { protocolo institucional, o que } \\
\text { gera um maior número de } \\
\text { complicações. }\end{array}$ \\
\hline $\begin{array}{l}\text { Prevalência e motivos de } \\
\text { remoção não eletiva do PICC em } \\
\text { neonatos }\end{array}$ & $\begin{array}{l}\text { Revista Gaúcha } \\
\text { de Enfermagem } \\
2012\end{array}$ & $\begin{array}{l}\text { Costa P; Kimura } \\
\text { AF; Vizzotto MPS; } \\
\text { Castro TE; West A; } \\
\text { Dorea E }\end{array}$ & $\begin{array}{l}\text { Estudo } \\
\text { transversal }\end{array}$ & $\begin{array}{l}\text { Os principais motivos para } \\
\text { remoção são: obstrução, } \\
\text { ruptura e edema do membro } \\
\text { cateterizado. }\end{array}$ \\
\hline
\end{tabular}

Fonte: elaboração dos autores.

\section{DISCUSSÃO}

A realização da revisão integrativa com a finalidade de sintetizar e ordenar os resultados obtidos sobre o tema possibilitou uma reflexão teórico-prática a respeito do papel do enfermeiro na utilização do cateter central de inserção periférica.

Considerando a "competência técnica e legal como pilar de sustentação na prática do enfermeiro com o PICC", foi constatado, em um estudo desenvolvido em cinco unidades públicas de tratamento intensivo neonatal, que $85,2 \%$ dos profissionais possuíam pós-graduação, porém $64,8 \%$ dos enfermeiros não eram habilitados para a inserção do cateter. Contudo, os melhores resultados em relação ao conhecimento da técnica de inserção, manutenção e retirada desse acesso venoso se deram justamente com os profissionais que possuíam habilitação para o PICC e que o inseriam rotineiramente ${ }^{1}$; o que reforça a importância do conhecimento teórico aliado à prática clínica para o sucesso na utilização do PICC pelos enfermeiros.

Em um estudo realizado em crianças em UTI neonatal e pediátrica, o uso do PICC foi citado em apenas 52\% das instituições. Dentre os motivos de não utilização, destacaram-se os aspectos relacionados ao conhecimento técnico-científico sobre o uso do cateter $(83,5 \%)^{2}$, o que remete novamente à necessidade de competência técnica para seu emprego.

A literatura aponta ainda que a utilização do PICC difunde-se cada vez mais, principalmente em UTI neonatal, ultrapassando em números o Cateter Venoso Central (CVC), tornando-se a primeira escolha, depois do cateterismo umbilical. Contudo, observa-se um tempo de permanência do dispositivo abaixo do esperado, ocasionado por perdas sucessivas devido a eventos adversos e complicações tais como obstrução, infiltração, suspeita de contaminação, 
tração, ruptura, edema do membro cateterizado e retirada acidental ${ }^{16-18}$. Todos esses eventos estão relacionados a problemas na prática clínica, que podem ser minimizados através da capacitação e educação permanente dos profissionais, visando a segurança do paciente ${ }^{16}$ o que evidencia que para que a competência técnica seja devidamente alcançada é necessário investimento na qualificação dos profissionais que manejam o PICC.

Sobre esta mesma vertente, foi identificado em um estudo que apenas $47,0 \%$ do total de enfermeiros entrevistados relatam ter aprendido o procedimento por meio de qualificação por sociedade de especialistas, revelando a necessidade de cursos, de caráter mais prático na formação acadêmica, a fim de capacitar novos enfermeiros para inserção do cateter ${ }^{2}$. Nesta compreensão, a qualificação e habilitação por sociedades ou cursos devidamente legalizados apontam para uma prática mais segura e com menos riscos.

Para além da competência técnica também é essencial que o enfermeiro paute suas ações na legalidade que envolve o uso do PICC. Nessa lógica, no conjunto de documentos legais adotados pelos enfermeiros, que visam padronizar condutas e melhorar a qualidade na assistência no manejo do PICC, destacam-se os protocolos institucionais, as normas, as rotinas e o Termo de Consentimento Informado, que desempenha o papel normalizador de um procedimento prescrito ${ }^{1-2}$.

Entretanto, um estudo revelou que de cinco unidades públicas de tratamento intensivo neonatal avaliadas, duas utilizavam o PICC rotineiramente, quatro possuíam protocolo para inserção, porém apenas $10 \%$ dos entrevistados referiram a existência desse documento ${ }^{1}$. Em outra pesquisa, a existência de protocolos para a inserção, manutenção e retirada do PICC mostrou-se predominante, onde a maior parte dos enfermeiros (58,5\%) afirmou utilizar formas específicas de registros relativos ao procedimento, porém, em relação ao Termo de Consentimento Informado (TCI), a maioria (94,2\%) revelou que não utiliza².

A de se destacar também a importância da criação de grupos de pesquisa, com trocas interinstitucionais, como forma de avaliar o desempenho em diferentes unidades de saúde, bem como a criação de times de terapia intravenosa. Tais ações conferem a adoção de boas práticas referentes ao procedimento, o que se apresentou de forma inconsistente ou pouco expressiva nas instituições que adotaram o cateter central de inserção periférica como dispositivo intravenoso de escolha ${ }^{2-7-16-17-19}$

Considerando "a prática clínica do enfermeiro na inserção, manutenção e retirada do PICC", destaca-se a localização do cateter como uma variável envolvida, visto que a mesma configura a certeza de que o dispositivo se localiza em um grande vaso, mais especificamente no terço médio da veia cava inferior ou superior, permitindo que fluidos infundidos através dela se diluam rapidamente ao sangue, diminuindo assim a possibilidade de complicações.
Um estudo revelou que o mau posicionamento inicial da ponta do cateter esteve relacionado ao superdimensionamento do comprimento da medida do cateter entre o sitio de inserção e a veia cava superior, o que exigiu a realização de manobras de extração, por parte do enfermeiro, para reposicionamento da ponta ${ }^{19}$. Isto aponta para a necessidade da revisão técnica da mensuração do comprimento do cateter, a fim de evitar o agravamento do quadro clínico do paciente, que pode ser letal nos casos de efusão pericárdica e de tamponamento secundário à perfuração miocárdica.

Outras complicações clínicas menos frequentes podem ser evitadas com essas ações, a exemplo da paralisia diafragmática devido à lesão do nervo frênico, ocasionada pelo extravasamento da nutrição parenteral, que tem como fatores predisponentes a migração inadvertida do cateter durante a troca de curativos, e a infecção ${ }^{20}$.

Nesta compreensão, apesar da redução de algumas complicações como hemorragia e sepse, o PICC apresenta maior incidência de mau posicionamento, resultando no surgimento de manifestações clínicas indesejáveis, tendo sua praticidade algumas vezes questionada quando é utilizado para infusões a longo prazo.

Ademais, complicações infecciosas têm sido associadas ao PICC quando a extremidade inferior é selecionada como local de inserção e quando ele é escolhido para a administração de NPT ${ }^{21}$.

Outros eventos adversos relacionados à utilização do PICC foram apontados na literatura científica, como: a dificuldade de progressão/fechamento da válvula capilar presenciados na fase de inserção do cateter, obstrução do cateter durante a sua manutenção e a ruptura/quebra do cateter durante a sua retirada ${ }^{22}$.

Portanto, apesar do PICC ser um dispositivo com muitas vantagens, as evidências atuais destacam a necessidade de uma equipe capacitada para sua inserção, manutenção e retirada, exigindo uma vigilância rigorosa através da visualização radiológica de sua localização. Pois, ainda são constatadas complicações diretas, relacionadas à própria anatomia e fisiologia do paciente, e indiretas, relacionadas à assistência prestada, incluindo o manejo inadequado do cateter ${ }^{3-17}$.

Diante dos fatos, uma equipe bem treinada mostra-se como critério importante e valoroso para a minimização na ocorrência de complicações, como infecções, obstruções e extravasamentos. A inserção e a manutenção seguras do cateter reduzem o risco de retirada antes do término do tratamento ${ }^{12}$.

E no que se refere à "tomada de decisão na prática clínica com o PICC", uma variedade de características e conceitos devem ser considerados, priorizados e mensurados pelo profissional responsável. Sendo assim, é necessário considerar os eventos adversos não intencionais, definidos como ocorrência com lesão resultante da atenção a saúde, os quais podem prolongar o tempo de permanência do paciente no hospital ou ocasionar o óbito ${ }^{22}$. 
O PICC se apresenta como um procedimento eficaz em muitos casos, como evidenciado em um relato de experiência sobre o seu uso em 24 crianças com diagnóstico de fibrose cística, submetidas ao tratamento prolongado por antimicrobianos, onde se obteve uma taxa de sucesso de $93,2 \%$ ao fim da terapêutica instituída ${ }^{23}$.

A tomada de decisão pela escolha do cateter PICC em relação ao CVC, foi observado em outro estudo realizado com pacientes oncológicos com profunda trobocitopenia sem agravos ou riscos adicionais. A análise prospectiva evidenciou alta taxa de sucesso, com excelente margem de segurança para complicações hemorrágicas e/ou necessidade de transfusões sanguíneas antes, durante ou imediatamente após o procedimento ${ }^{24}$.

Conlusão semelhante foi obtida através de um estudo ${ }^{25}$ que comparou a praticidade e efetividade do PICC com relação ao CVC no tratamento de crianças com câncer, revelando que o PICC, além de proporcionar acesso intravenoso de longo prazo confiável em crianças que sofrem dessa patologia, reduz a dor e minimiza o estresse psicológico, melhorando a qualidade de vida durante o tratamento quimioterápico.

Outra evidência científica com relação ao uso do PICC foi observada no tratamento de pacientes com linfomas e leucemias, onde foi constatado que cerca de $7,8 \%$ dos pacientes (39 de 498) apresentava trombose venosa profunda (TVP) sintomática associada ao PICC quando localizados na veia subclavia, axilar, braquial e veia jugular. Considerando o resultado, o protocolo de inserção da instituição, foi modificado, onde o PICC passou a ser inserido na veia jugular interna sob orientação ecográfica com local de saída direcionado na região subclavicular, obtendo, como resultado dessa tomada de decisão, uma considerável redução na incidência de trombose venosa profunda relacionada ao uso do $\mathrm{PICC}^{26}$.

Ademais, na tomada de decisão relacionada ao PICC, a orientação radiológica é uma aliada, tendo em vista que taxa de sucesso passa de 74 para $100 \%$ quando a análise radiológica é realizada ${ }^{27}$.

Além disso, o PICC tem se mostrado mais vantajoso ao CVC nas diversas especialidades de atenção à saúde, inclusive na oncologia ${ }^{24-26}$, pois possibilita minimizar o risco de traumas e de sangramento relacionado ao procedimento, mesmo em pacientes oncológicos com quadro de trombocitopenia grave ${ }^{24}$.

\section{CONCLUSÃO}

As evidências científicas apontam o importante papel do enfermeiro na utilização do cateter central de inserção periférica tanto na neonatologia quanto na pediatria, e que seu uso tem sido crescente nos contextos da terapia intensiva da oncologia.

Todavia, eventos adversos ainda se mostram frequentes, o que torna imprescindível aliar a prática clínica com o
PICC à capacitação, à habilitação e ao treinamento dos profissionais, além da importância da criação de times de terapia intravenosa como forma de minimizar potenciais complicações.

Ademais sugere-se o desenvolvimento de documentos institucionais como normas, rotinas, protocolos e termos de consentimento informado, com o objetivo padronizar condutas e sustentar legalmente essa prática.

Apesar de o estudo ter alcançado o objetivo proposto, este possui limitações devido ao número restrito de artigos acerca do tema. Por outro lado, esta pesquisa possui como potencialidade a síntese do conhecimento cientifico mais atualizado sobre a prática clínica com o PICC.

\section{REFERÊNCIAS}

1 Belo MPM, Silva RAMC, Nogueira ILM, Mizoguti DP, Ventura CMU. Conhecimento de enfermeiros de Neonatologia acerca do Cateter Venoso Central de Inserção Periférica. Rev Bras Enferm, Brasília. 2012; 65(1): 42-8.

2 Vendramim P, Pedreira MLG, Peterlini MAS. Cateteres centrais de inserção periférica em crianças de hospitais do município de São Paulo. Rev Gauch Enferm. 2007; 28(3):331-9.

3 Camara D. Minimizing risks associated with peripherally inserted central catheter in the NICU. Am J Mater Child Nurs. 2001; 26(1):17-21.

4 Toma E. Avaliação do uso do PICC cateter central de inserção periférica em recém-nascidos - tese de Doutorado. São Paulo: Escola de Enfermagem, Universidade de São Paulo. 2004; 145 f.

5 Vendramin P. Cateter central de inserção periférica (CCIP). In: Harada MJCS, Rego RC, Orgs. Manual de terapia intravenosa em pediatria. São Paulo: ELLU. 2005; 75-95.

6 Conselho Federal de Enfermagem. Resolução $n^{\circ}$ 258, 12 de julho de 2001 [Internet]. Dispõe sobre a Inserção do Cateter Periférico Central Por enfermeiros. [Acesso em 10 ago 2015]. Disponível em: http://www.cofen.gov.br/resoluocofen-2582001_4296.html.

7 Dórea E, Castro TE, Costa PKAF, Santos FMG. Práticas de manejo do cateter central de inserção periférica em uma unidade neonatal. Rev Bras Enferm. 2011; 64(6): 997-1002.

8 Chlebicki MP, Teo EK. Review of peripherally inserted central catheters in the Singapore acute-care hospital. Singapore Med J. 2003; 44(10):531-5.

9 Pettit J, Wyckoff MM. Peripherally inserted central catheters: guideline for practice. 2nd ed. Glenview: National Association of Neonatal Nurses, [Internet]. 2007 [Acesso em 15 ago 2015]. Disponível em: http://www.nann.org/pdf/PICCGuidelines.pdf

10 Griffiths VR, Philpot P. Peripherally inserted central catheters (PICCS): do they have a rolle in the care of the critically ill patient? Intensive Crit Care Nurs 2002; 18(1):137-47.

11 Jesus VC, Secoli SR. Complicações acerca do cateter venoso central de inserção periférica (PICC). Cienc Cuid Saude. 2007; 6(2): 252-260.

12 Rodrigues ZS, Chaves EMC, Cardoso MVLML. Atuação do enfermeiro no cuidado com o cateter central de inserção periférica no recém-nascido. Rev bras enferm. 2006; 59(5): 626-9. 
13 Conselho Federal de Enfermagem. Lei 7.498, de 25 de junho de 1986. Lei do Exercício Profissional de Enfermagem [Internet]. 1986 [Acesso em 12 set 2015]. Disponível em: http://www. planalto.gov.br/ccivil_03/leis/l7498.htm.

14 Conselho Federal de Enfermagem. Resolução n² 258, 12 de julho de 2001. Dispõe sobre a Inserção do Cateter Periférico Central Por enfermeiros [Internet]. 2001 [Acesso em 12 set 2015]. Disponível em: http://www.cofen.gov.br/resoluocofen-2582001_4296.html.

15 Santos El. Cuidado e prevenção das skin tears por enfermeiros: revisão integrativa de literatura. Rev Gaúcha Enferm. 2014; 35(2): 142-9.

16 Baggio MA, Bazzi FCS, Bilibio CAC. Cateter central de inserção periférica: descrição da utilização em UTI Neonatal e Pediátrica. Rev Gaúcha Enferm. 2010; 31(1):70-76.

17 Costa $P$, et al. Prevalência e motivos de remoção não eletiva do cateter central de inserção periférica em neonatos. Rev Gaucha Enferm. 2012; 33(3):126-133.

18 Shah PS, Shah VS. Continuous heparin infusion to prevent thrombosis and catheter occlusion in neonates with peripherally placed percutaneous central venous catheters. Cochrane Database Syst Rev. 2008; 16 (2):CD002772.

19 Camargo PP, Kimura AF, Toma E, Tsunechiro MA. Localização inicial da ponta de cateter central de inserção periférica (PICC) em recém-nascidos. Rev Esc Enferm USP. 2008; 42(4):723-8.

20 Toselloa B, et al. Hemidiaphragmatic paralysis in preterm neonates: a rare complication of peripherally inserted central catheter extravasation. J Pediatr Surg. 2011; 46(7)17-21.

21 Advani S, Reich NG, Sengupta A, Gosey L, Milstone AM. Central Line-Associated Bloodstream Infection in Hospitalized Children with Peripherally Inserted Central Venous Catheters: Extending Risk Analyses Outside the Intensive Care Unit. Clin Infect Dis. 2011; 52(9):1108-1115.

22 Sá JS, Bezerra ALQ, Tobias GC, Paranaguá TTB. Eventos adversos na utilização do cateter central de inserção periférica em hospital público.J Nurs UFPE. 2015; 9(8): 8802-9.

23 Buia S, et al. Intravenous peripherally-inserted central catheters for antibiotic therapy in children with cystic fibrosis. J Cyst Fibros. 2009; 8(5): 326-31.

24 Potet J, et al. Peripherally inserted central catheter placement in cancer patients with profound thrombocytopaenia: a prospective analysis. European Radiology. 2013; 23(7): 2042-8.

25 Hatakeyama N, et al. An evaluation of peripherally inserted central venous catheters for children with cancer requiring long-term venous access. Int J Hematol. 2011; 94(4):372-7.

26 Periard D. Peripherally inserted central catheter in leukemia: insertion site determines clotting risk. Leukemia and lymphoma. Leuk Lymphoma. 2010; 51(8):1391-2.

27 Amerasekera SSH; Jones CM; Patel R; Cleasby MJ. Imaging of the complications of peripherally inserted central venous catheters. Clin Radiol. 2009; 64(8):832-40. 